\title{
Impact of preventive screening and lifestyle interventions in women with a history of preeclampsia: A micro-simulation study
}

European Journal of Preventive

\author{
GR Lagerweij ${ }^{1,2}$, L Brouwers ${ }^{2,3}$, GA De Wit ${ }^{1,4}$, KGM Moons', \\ L Benschop ${ }^{2,5}$, AHEM Maas ${ }^{6}$, A Franx ${ }^{3}$, MJH Wermer ${ }^{8}$, \\ JE Roeters van Lennep ${ }^{5}$, BB van Rijn ${ }^{3}$ and $H$ Koffijberg ${ }^{1,7}$; \\ on behalf of the CREW Consortium
}

\begin{abstract}
Background: Preeclampsia is a female-specific risk factor for the development of future cardiovascular disease. Whether early preventive cardiovascular disease risk screenings combined with risk-based lifestyle interventions in women with previous preeclampsia are beneficial and cost-effective is unknown.

Methods: A micro-simulation model was developed to assess the life-long impact of preventive cardiovascular screening strategies initiated after women experienced preeclampsia during pregnancy. Screening was started at the age of $\mathbf{3 0}$ or $\mathbf{4 0}$ years and repeated every five years. Data (initial and follow-up) from women with a history of preeclampsia was used to calculate 10-year cardiovascular disease risk estimates according to Framingham Risk Score. An absolute risk threshold of $2 \%$ was evaluated for treatment selection, i.e. lifestyle interventions (e.g. increasing physical activity). Screening benefits were assessed in terms of costs and quality-adjusted-life-years, and incremental cost-effectiveness ratios compared with no screening.

Results: Expected health outcomes for no screening are 27.35 quality-adjusted-life-years and increase to 27.43 qualityadjusted-life-years (screening at 30 years with $2 \%$ threshold). The expected costs for no screening are $€ 9426$ and around $€ 13,88 \mathrm{I}$ for screening at 30 years (for a $2 \%$ threshold). Preventive screening at 40 years with a $2 \%$ threshold has the most favourable incremental cost-effectiveness ratio, i.e. $€ 34,996 /$ quality-adjusted-life-year, compared with other screening scenarios and no screening.

Conclusions: Early cardiovascular disease risk screening followed by risk-based lifestyle interventions may lead to small long-term health benefits in women with a history of preeclampsia. However, the cost-effectiveness of a lifelong cardiovascular prevention programme starting early after preeclampsia with risk-based lifestyle advice alone is relatively unfavourable. A combination of risk-based lifestyle advice plus medical therapy may be more beneficial.
\end{abstract}

\section{Keywords}

Cardiovascular disease prevention, cost-effectiveness, lifestyle intervention, primary prevention, preeclampsia

Received 21 June 2019; accepted 10 December 2019

\footnotetext{
'Julius Center for Health Sciences and Primary Care, University Medical Center Utrecht, the Netherlands

${ }^{2}$ Netherlands Heart Institute, the Netherlands

${ }^{3}$ Wilhelmina Children's Hospital Birth Center, University Medical Center Utrecht, the Netherlands

${ }^{4}$ Centre for Nutrition, Prevention and Healthcare, National Institute for Public Health and the Environment, the Netherlands

${ }^{5}$ Department of Obstetrics and Gynecology, Erasmus MC, the Netherlands

${ }^{6}$ Department of Cardiology, Radboud University Medical Center, the Netherlands
}

\footnotetext{
${ }^{7}$ Department of Health Technology and Services Research, University of Twente, the Netherlands

${ }^{8}$ Department of Neurology, Leiden University Medical Center, the Netherlands

*Full author list available in the Acknowledgements.
}

Corresponding author:

Giske Lagerweij, Julius Center for Health Sciences and Primary Care, University Medical Center Utrecht, Stratenum 6.I3I, P.O. Box 85500, 3508 GA Utrecht, the Netherlands. Email: g.r.lagerweij@umcutrecht.nl 


\section{Introduction}

Cardiovascular disease (CVD) is the most prevalent cause of death in women worldwide. ${ }^{1}$ The global burden of CVD is associated with lifelong exposure to traditional risk factors, such as hypertension, obesity, smoking and diabetes and is strongly associated with a prolonged unhealthy lifestyle. ${ }^{1,2}$ It has been estimated that up to $90 \%$ of CVD risk can be explained through traditional and modifiable risk factors. ${ }^{3}$ Over the past decades, long-term population studies have identified preeclampsia as one of the strongest female-specific risk factors for CVD, associated with a two- to sevenfold increased risk of developing ischaemic heart disease and stroke compared to women with normotensive pregnancies. ${ }^{4-10}$

Several international obstetric guidelines recommend screening for cardiovascular risk factors in women with a history of preeclampsia at the age of 50 years. ${ }^{11-13}$ Leading cardiovascular prevention guidelines, however, have not yet sufficiently implemented these recommendations. ${ }^{14-17}$ Additionally, clinically used treatment recommendations are based on risk-prediction models that calculate 10-year CVD risk which are strongly age-dependent. Shortly after pregnancy, women will often not reach the current risk threshold for preventive measures recommended by these guidelines. For example, in women with mean age of 31 years (standard deviation (SD) 4.5), the average 10-year CVD risk according to the Framingham Global Risk score is $1.08 \%(95 \%$ confidence interval $(\mathrm{CI})$ of $1.04-1.12 \%)$ whereas the recommended risk threshold is $10 \%{ }^{18}$ Current risk-based selection may therefore not be appropriate for these young women at relatively high but low absolute risk and a lifetime CVD risk-based approach may be preferable. ${ }^{19}$

As the timeline during which benefits from preventive intervention in young women accrue is lengthy, a randomised or cohort setting is not feasible to assess the full benefits of prevention. Here, a model-based approach is valuable, even though collecting the required evidence is challenging. Two Dutch Markov model-based studies previously showed that early CVD prevention in women with previous preeclampsia is likely to be costeffective. $^{20,21}$ However, authentic long-term follow-up data from cardiovascular screening including multiple cardiovascular risk factor measures for each participant were not available at the time these studies were performed. Furthermore, previous studies used a cohort model that is not able to include treatment decisions on an individual level, which is likely to give a less realistic representation of clinical practice.

We present a model-based patient-level simulation (i.e. micro-simulation) of early cardiovascular risk screening combined with risk-based lifestyle interventions to assess health benefits, costs and costeffectiveness in women with a history of preeclampsia. We incorporated individual patient data on cardiovascular risk factor measures, e.g. blood pressure level, cholesterol level and smoking status, of an initial cardiovascular screening six months after delivery in women with preeclampsia and of screening after 10-20 years follow-up to estimate 10-year CVD risks. Given the young age and the low absolute CVD risk, it is unlikely that initial pharmaceutical treatment is acceptable, therefore we focused on the benefits of lifestyle interventions. A life-long horizon was applied to capture all benefits of screening and subsequent lifestyle interventions in these women.

\section{Methods}

A discrete time micro-simulation model was developed to assess the impact of early preventive strategies for $\mathrm{CVD}^{22}$ The flowchart of this model is presented in Supplemental Material Appendix A. The time cycle of the model was one year. Women were followed until death and outcomes were aggregated at population level, i.e. total CVD events, total costs and health outcomes, expressed in quality-adjusted-life-years (QALYs). Supplemental Material Appendix B shows an overview of all input parameters that were used in the analysis. Estimates for model parameters were based on evidence from the literature and partially on expert opinion and consensus. Relatively wide distributions were used to properly reflect any parameter uncertainty.

In total, we simulated a hypothetical cohort of 2000 women as the incidence of early-onset preeclampsia is currently about $1-2 \%$ amongst a total of approximately 171,000 annual pregnancies in the Netherlands. ${ }^{23,24}$ Women entered the simulation model at the average age of a first pregnancy in the Netherlands (i.e. at 30 years old). ${ }^{23}$

\section{CVD risk estimates}

As CVD risk estimates vary with age, we assumed that CVD risk increased over time for each woman. Published long-term data on the development of risk factors was not available for this specific group of women with previous preeclampsia. Therefore, we used data from two studies in the Netherlands to calculate 10-year CVD risk estimates. More information on the used datasets can be found in Supplemental Material Appendix C. Both studies measured cardiovascular risk parameters at different time intervals after preeclampsia.

The Framingham Global Risk Score (FRS) was used to estimate 10-year CVD risk at initial post-partum 
screening and at follow-up. ${ }^{25}$ Multiple imputation (with 10 datasets) was performed to handle missing predictor data using the MICE package in $\mathrm{R}^{26}$ Imputation was based on all other available patient characteristics, such as age, sex, blood pressure and cholesterol levels.

Estimated CVD risk estimates and follow-up time were not the same for all women in the two cohorts due to differences in age at screening in both studies. To correct for this, 10-year CVD risk estimates were recalculated to risk estimates at the same age (for more details see Supplemental Material Appendix D).

\section{Usual care}

Despite a national multidisciplinary guideline recommending that women who experienced preeclampsia should be offered CVD screening by their general practitioner at the age of 50 years, no nationwide primary prevention programme is currently offered in the Netherlands. ${ }^{16}$ We therefore assumed usual care for these women as follows. We presumed that annually $3 \%$ (range $2-4 \%$ ) of all women above the age of 60 years would undergo a cardiovascular screening and could then be identified as high-risk. Usual care applied a risk threshold of $10 \%$ (FRS) to classify women as high risk. ${ }^{27,28}$ Lifestyle interventions (including smoking cessation, weight reduction, increasing physical activity) were recommended to high-risk women as preventive intervention. Medication was not used as preventive intervention due to the young age of the women. For those women adhering to these lifestyle change, we used risk reduction (average 0.91, range 0.84-0.96) in the model. ${ }^{29}$ Finally, because evidence on long-term adherence rates was not available, we assumed that, on average, $20 \%$ of women stayed adherent up to 10 years after initiation of the intervention and derived the annual adherence rate through exponential interpolation.

\section{Preventive strategy for CVD}

The CVD prevention strategies for women after their preeclampsia were defined as cardiovascular risk screening starting at the age of 30 or 40 years, with screening repeated every five years and ending at the age of 55 years, followed by lifestyle advice based on these risks. As women were young at enrolment in the model, the current recommended generic risk threshold (FRS $>10 \%$ ) was too high, yielding hardly any women in the high-risk category. We therefore had to apply lower risk thresholds for the purpose of this study (i.e. FRS $>2 \%$ and $>5 \%$ ). We used the response rate of the women invited to participate in the follow-up study to estimate the proportion that would participate in such a screening programme $(39 \%$, range $21-60 \%)$. Women who had already experienced CVD (with one or more CVD event(s)) were not considered eligible for (primary) preventive screening, but remained in the micro-simulation, potentially experiencing sequential CVD events, until they died. Women who were assessed as low-risk at the previous screening or who did not adhere to the lifestyle changes were invited to the subsequent screening event(s) after five years.

Lifestyle interventions (including smoking cessation, weight reduction, increasing physical activity) were the recommended preventive intervention for women classified as high risk (i.e. FRS $>2 \%$ and $>5 \%$ ), consistent with usual care. As data on adherence was lacking, we assumed that the relative change that younger women were adherent to lifestyle interventions was equal to the 10 -year adherence of $20 \%$ in older women (see usual care). However, given uncertainty regarding this adherence rate a relative change of 0.9 (lower) to 1.1 (higher) to this $20 \%$ adherence was used to define a plausible range of values.

\section{Model parameters}

All model parameters are provided in Supplemental Material Appendix B. Three CVD event categories are distinguished in this study; coronary heart disease (CHD), stroke, and other cardiovascular disease (OCVD) events. The CVD events could be either fatal or non-fatal, resulting in incorporation of six total CVD event types. The relative occurrence of the six event types was age-dependent and based on previous literature (Supplemental Material Appendix B Table 2). ${ }^{19,30,31}$ When a cardiovascular event occurred, the CVD risk estimate was proportionally increased (relative risk ratio 2.1, range 1.7-2.6).

Although women may experience other outcomes, (e.g. mental or psychosocial problems) after preeclampsia, little follow-up data are available regarding these long-term outcomes and their effects (and relevance) on quality of life $(\mathrm{QoL})$. $^{32,33}$ Therefore, we used QoL values (utilities) available for women from the general population and adjusted for age. ${ }^{34,35}$

QoL was proportionally reduced after the occurrence of a CVD event. ${ }^{34,36-38}$ The proportional reduction in QoL after a first CVD event depended on the CVD event type, but remained the same for similar recurrent CVD events. Also, the decrease in QoL after a CVD event was lower in the first year compared to consecutive years after the event. It was assumed that women with a CVD event would receive medication. Side-effects of prescribed medication after a CVD event were assumed to be incorporated in the disutility of the event and therefore not taken into account separately in the analysis.

Dutch studies and evidence from National Institute for Health and Care Excellence were used for the 
estimation of the costs of CVD events. ${ }^{34,36-38}$ Similar to the utilities, costs varied over the six different CVD types. Costs for recurrent events were assumed to be similar to costs for first-time events. Costs of the first year after a CVD event were set higher than costs the subsequent years. Costs of the screening programme included a visit to the general practitioner and laboratory tests, and were applied to all women who participated in the screening programme. Costs of preventive lifestyle interventions were applied to all women who were classified as high-risk, i.e. women with CVD risk estimates that exceeded the intervention threshold of $2 \%$ and $5 \%$, regardless of their adherence to these lifestyle interventions.

An overview of all utilities and costs together with the distribution for the sensitivity analyses is presented in Supplemental Material Appendix B Table 1 (rows 14-44). Following Dutch guidelines, a discount rate of $4 \%$ for costs and $1.5 \%$ for health outcomes was applied. ${ }^{39}$ As preventive screening, CVD events and death due to natural causes can occur at any time during the year (instead of only at the start or end of a year) a half-cycle correction was applied in the model.

\section{Cost-effectiveness analysis}

The cost-effectiveness analysis was performed with the incremental cost-effectiveness ratio (ICER) as outcome, using a healthcare perspective. This ratio represents the difference in lifetime costs divided by the difference in effectiveness, i.e. health outcomes. The difference in costs and effectiveness is defined as the difference between the four preventive strategy (i.e. screening starting at different age (i.e. 30 and 40 years) and risk levels (i.e. $2 \%$ and $5 \%$ ), with subsequent lifestyle interventions) and usual care. Probabilistic sensitivity analysis was applied to assess how uncertainty in parameter values resulted in uncertainty in the effect and cost outcomes. To determine the differences between strategies, we used 4500 Monte-Carlo simulations applied to a cohort of 2000 hypothetical, unique women. Furthermore, the probability of a preventive screening to be cost-effective compared to alternative strategies and usual care was estimated as a function of the willingness-to-pay (WTP) and presented in cost-effectiveness-acceptability curves. A commonly applied Dutch WTP threshold of $€ 20,000$ per QALY gained is used to determine whether a screening strategy is cost-effective or not, and to calculate the incremental net health benefits (INHBs).

Lastly, we performed a value of information (VOI) analysis to investigate the value of collecting additional information on the parameters used to reduce the uncertainty in cost-effectiveness outcomes. We used the Sheffield Accelerated Value of Information (SAVI) tool to estimate the expected value of perfect information
(EVPI) and expected value of partial perfect information (EVPPI). ${ }^{40}$ The value of hypothetically resolving all uncertainty is reflected by the EVPI whereas the EVPPI indicates what the value is of resolving all uncertainty in one parameter or a group of parameters. ${ }^{41}$

\section{Results}

Supplemental Material Appendix C shows the baseline table of the two cohorts and the number of missing data. Supplemental Material Appendix E shows the authentic risk estimates of the women included in both cohorts.

\section{Cost-effectiveness analysis}

Table 1 shows the results of the cost-effectiveness analysis using the chosen risk thresholds of $2 \%$ and $5 \%$. No screening has slightly lower health outcomes and costs compared to all four preventive screening scenarios, i.e. 27.35 QALYs and $€ 9426$ per woman. Screening scenarios starting at 40 years have similar health benefits (27.41 QALYs) and the scenario with a $5 \%$ threshold has slightly higher costs $(€ 11,578$ versus $€ 11,561)$. Screening starting at 30 years with a $2 \%$ threshold has comparable health effects $(27.42$ versus 27.43 QALYs) and higher costs (€13,881 versus $€ 13,078)$ than with a $5 \%$ threshold.

When comparing the screening strategies with each other, preventive screening starting at 40 years and with a $2 \%$ threshold is the 'favourable' preventive screening in terms of the ICER, i.e. $€ 34,996 /$ QALY. Although screening starting at 40 years with a $5 \%$ threshold is less costly, it has less health benefits resulting in a slightly higher ICER. Therefore, screening starting at 40 years with a $5 \%$ threshold is dominated by screening starting at 40 years with a $2 \%$ threshold. However, the latter strategy would not be considered cost-effective if a WTP threshold of $€ 20,000 /$ QALY is applied.

Screening starting at 30 years with a $5 \%$ threshold is the second 'best' screening strategy in terms of costeffectiveness; the ICER is $€ 101,092 / \mathrm{QALY}$, compared with screening starting at 40 years with a $2 \%$ threshold. Screening starting at 30 years with a $2 \%$ threshold is dominated by preventive screening starting at 30 years with a $5 \%$ threshold due to similar health benefits but slightly higher costs. Screening starting at 40 years with a $5 \%$ threshold and screening starting at 30 years with a $2 \%$ threshold are therefore dominated by other strategies (see strikethrough in Table 1).

Figure 1 shows the average cost-effectiveness plane with the average health effects and costs for the four preventive screening scenarios. Furthermore, it shows the incremental cost-effectiveness plane where scenario B (preventive screening starting at 40 years with a $2 \%$ 
Table I. Impact (costs and health benefits) of different scenarios for cardiovascular screening every five years and lifestyle interventions in women with previous preeclampsia.

\begin{tabular}{|c|c|c|c|c|c|c|c|c|}
\hline & & $\begin{array}{l}\text { Average } \\
\text { costs }(€)\end{array}$ & $\begin{array}{l}\text { Average } \\
\text { health } \\
\text { benefits } \\
\text { (QALY) }\end{array}$ & & & & & \\
\hline \multicolumn{2}{|c|}{ No screening } & 9426 & 27.35 & & & & & \\
\hline \multicolumn{3}{|c|}{ Preventive screening } & & Comparing & $\begin{array}{l}\text { Incremental } \\
\text { cost }(€) \\
(95 \% \mathrm{Cl})\end{array}$ & $\begin{array}{l}\text { Incremental health } \\
\text { benefits } \\
\text { (QALY) }(95 \% \mathrm{Cl})\end{array}$ & $\begin{array}{l}\text { ICER } \\
(€ / Q A L Y)\end{array}$ & INHB \\
\hline$A$ & $\begin{array}{l}\text { Screening starting } \\
\quad 40+5 \% \text { threshold }\end{array}$ & I I,578 & 27.41 & $\begin{array}{l}\text { A to } 0^{\mathrm{a}} \\
\text { (Dominated by } \mathrm{B})\end{array}$ & $\begin{array}{l}z 15 z \\
(168-4394)\end{array}$ & $\begin{array}{l}0.06 \\
(+.57+.84)\end{array}$ & 37,098 & -0.05 \\
\hline$B$ & $\begin{array}{l}\text { Screening starting } \\
\qquad 40+2 \% \text { threshold }\end{array}$ & $|I, 56|$ & $27.4 I$ & $\mathrm{~B}$ to $0^{\mathrm{a}}$ & $\begin{array}{l}2135 \\
(-378-4405)\end{array}$ & $\begin{array}{l}0.06 \\
(-1.7|-| .80)\end{array}$ & 34,996 & -0.05 \\
\hline$C$ & $\begin{array}{l}\text { Screening starting } \\
\quad 30+2 \% \text { threshold }\end{array}$ & $|3,88|$ & 27.42 & $\begin{array}{l}C \text { to } B \\
\text { (Dominated by } D)\end{array}$ & $\begin{array}{l}2320 \\
(325-5170)\end{array}$ & $\begin{array}{l}0.0+ \\
(+1.72+1,76)\end{array}$ & 210,894 & -0.15 \\
\hline$D$ & $\begin{array}{l}\text { Screening starting } \\
\quad 30+5 \% \text { threshold }\end{array}$ & 13,078 & 27.43 & $D$ to $B$ & $\begin{array}{l}1526 \\
(-1134-4363)\end{array}$ & $\begin{array}{l}0.01 \\
(-1.68-1.77)\end{array}$ & 101,092 & -0.11 \\
\hline
\end{tabular}

ICER: incremental cost-effectiveness ratio; INHB: incremental net health benefit; QALY: quality-adjusted-life-year.

The table is organized following the principles of the cost-effectiveness frontier: the investigated interventions are sorted based on the effectiveness and compared amongst each other. In other words, each intervention is compared with the next best effective intervention. An intervention can be ruled out if another intervention (or usual care) is both more effective and less costly. We have chosen to show all four interventions and strike through the interventions that are ruled out (i.e. dominated) by another intervention.

${ }^{\mathrm{a}} \mathrm{S}$ cenario 0 represents usual care.

threshold) is compared with no screening (scenario 0 ), and preventive screening starting at 30 years with a $5 \%$ threshold (scenario D). The dotted line in Figure 1 is the WTP threshold of $€ 20,000 /$ QALY. The probabilistic sensitivity analysis (PSA) samples of the two scenarios are almost similar.

Figure 2 shows the cost-effectiveness acceptability curve. For a WTP threshold of $€ 20,000 /$ QALY, no screening has the largest probability of being cost-effective, i.e. probability of $72 \%$. For a WTP threshold above $€ 57,000 /$ QALY all screening strategies are more likely to be cost-effective than no screening, but no single strategy clearly outperforms the other strategies.

\section{VOI analysis}

Table 2 shows the main results from the VOI analysis. The VOI analysis indicates that the (annual) overall EVPI per person affected is $€ 5023$ per person, or 0.25 QALYs per person. Furthermore, the analysis indicates that the average single parameter 10 -year CVD risk at 30 years has the largest EVPI value per person (i.e. $€ 1568)$. Further investigation of the uncertainty of single parameters shows that collecting more information on the average 10-year CVD risk for older women, cost of a stroke in the first year and treatment effectiveness has added value (see Table 2, rows 2-4). Given that 2000 women present with previous preeclampsia in the Netherlands per year, the population EVPI equals $€ 10.1 \mathrm{~m}$ per year.

Supplemental Material Appendix G Table 1 shows an overview of the groups of associated parameters that are used to estimate the group EVPPI. Collecting additional information on parameters related to predicted CVD risk (i.e. set 1), has the largest value with an EVPPI of $€ 1696$ per person (Table 2, rows 8-10). Supplemental Material Appendix G Table 2 shows the results for the VOI analysis on all single parameters (rows 2-28) and group parameters (rows 31-36).

\section{Discussion}

\section{Short summary of findings}

In this simulation study, we found that early (i.e. starting at 30 or 40 years old) and repeated (every five years) CVD risk screening and risk-based lifestyle interventions after preeclampsia potentially reduces CVD risk and improves health outcomes. However, preventive CVD risk screening and risk-based lifestyle intervention alone with an absolute risk threshold of $2 \%$ or $5 \%$ are not cost-effective.

\section{Clinical implications}

Although our model estimates that early CVD risk screening and risk-based lifestyle interventions may 


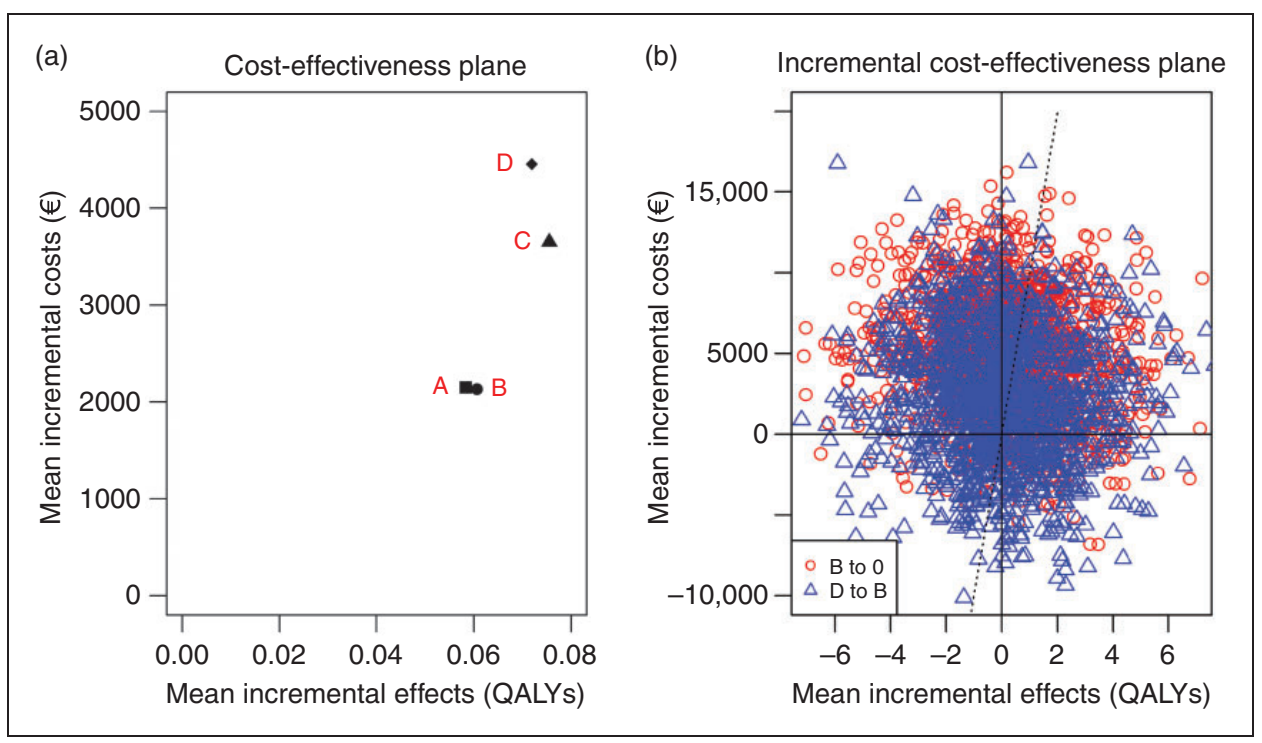

Figure I. Results of the probabilistic sensitivity analyses (PSAs). The average health benefits and costs are shown in the costeffectiveness plane for all four screening scenarios (a). The difference in benefits and costs of promising screening scenarios are shown in the incremental cost-effectiveness plane with a willingness-to-pay (WTP) threshold of $€ 20,000 / q u a l i t y$-adjusted-life-year (b).

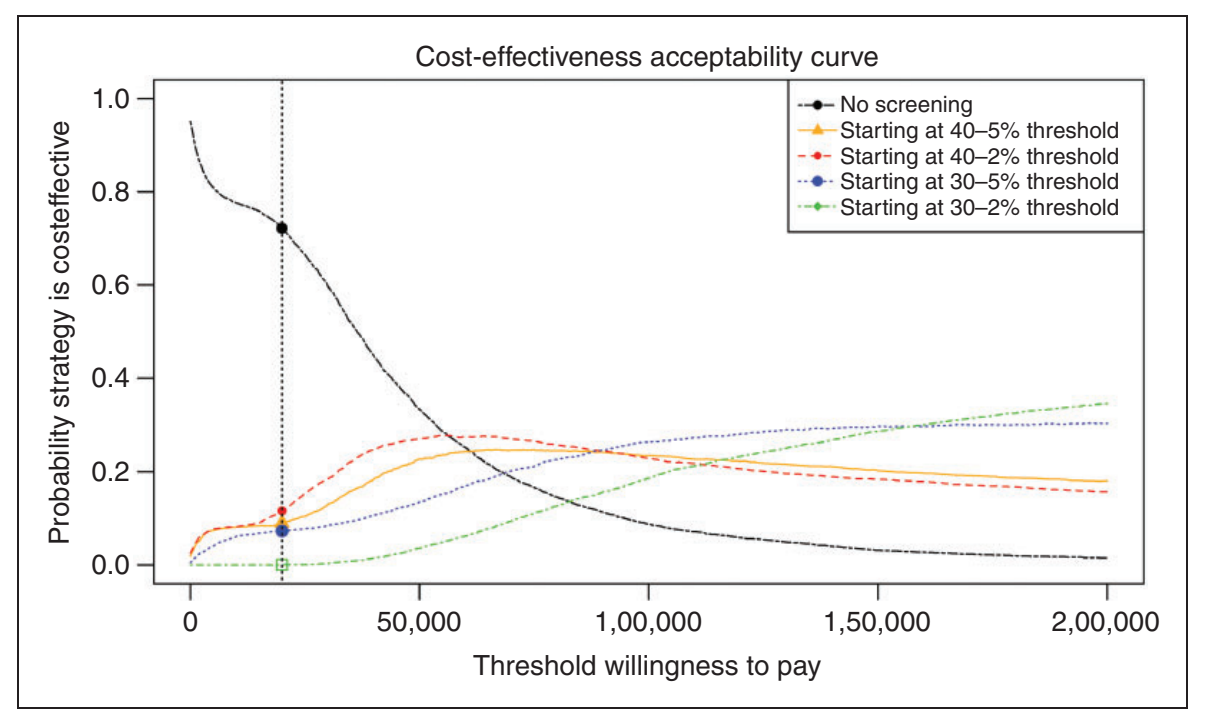

Figure 2. Cost-effectiveness acceptability curves for preventive screening and lifestyle interventions in women with a history of preeclampsia.

lead to very small health benefits, it is not cost-effective (with the current model settings). Some of the following aspects need to be considered for implications in clinical practice. In our experience, offering cardiovascular screening to women after (especially early-onset) preeclampsia results in relatively high percentage of women willing to participate. Unfortunately, the current cardiovascular screening for these women takes place in the hospital which may result in a lower participation rate of these women, i.e. mothers with young children who do not attend the half day of in-hospital screening. Although specific risk factors, such as familiar hypercholesterolaemia, should be treated by a vascular specialist, implementation of screening and lifestyle interventions in Dutch primary care would be more efficient. The Dutch general practitioner (GP) system is well structured and easily accessible, but such a system may not be available in some other countries.

In addition, our model only included the health effects gained by reducing cardiovascular outcomes. It 
Table 2. Summary of main results from value of information analysis (see Supplemental Material Appendix F Table 2 for the complete set of results).

\begin{tabular}{|c|c|c|c|c|c|}
\hline & $\begin{array}{l}\text { Per person } \\
\text { EVPPI }(€)\end{array}$ & $\begin{array}{l}\text { Standard } \\
\text { error }\end{array}$ & $\begin{array}{l}\text { Indexed } \\
\text { to overall } \\
\mathrm{EVPI}=1.00\end{array}$ & $\begin{array}{l}\text { EVPPI for } \\
\text { Netherlands } \\
\text { per year }(€)\end{array}$ & $\begin{array}{l}\text { EVPPI for } \\
\text { Netherlands } \\
\text { over } 5 \text { years }(€)\end{array}$ \\
\hline \multicolumn{6}{|l|}{ Single parameter EVPPI } \\
\hline Average 10 -year CVD risk at age 30 & 1567.6 & 129.4 & 0.3 & $3,135,000$ & $15,680,000$ \\
\hline Average I0-year CVD risk at age 80 & 259.7 & 98.1 & 0.1 & 519,400 & $2,597,000$ \\
\hline Cost of stroke event (first year) & 44.3 & 40.1 & 0.0 & 88,630 & 443,100 \\
\hline $\begin{array}{l}\text { Relative risk of CVD with preventive } \\
\text { intervention versus without intervention }\end{array}$ & 21.0 & 37.1 & 0.0 & 41,930 & 209,700 \\
\hline Utility of other CVD event (sequential year) & 12.2 & 37.0 & 0.0 & 24,300 & $|2|, 500$ \\
\hline \multicolumn{6}{|l|}{ Group parameter EVPPI } \\
\hline $\begin{array}{l}\text { Set I - predicted CVD risk } \\
\text { The following parameters were grouped: } \\
\text { average } 10 \text {-year CVD risk at } 30 \text { years; } \\
\text { average } 10 \text {-year CVD risk at } 80 \text { years; } \\
\text { marginal correlation between risk } \\
\text { profiles (per } 10 \text { years). }\end{array}$ & 1696.3 & 134.3 & 0.3 & $3,392,611$ & $16,963,053$ \\
\hline
\end{tabular}

CVD: cardiovascular disease; EVPI: expected value of perfect information; EVPPI: expected value of partial perfect information.

is likely that the proposed lifestyle interventions (i.e. weight reduction, smoking cessation and improving physical activity patterns) have an additional health benefit in preventing other (non-cardiovascular) health problems, such as preventing joint problems in obese patients and chronic pulmonary problems in smokers. Additionally, the lowering of risk factors will likely reduce risk of other long-term events, such as hypertension and subsequent renal failure, which were currently not incorporated in the model. This may result in further lengthening of life in good health as women age. Taking the facts together we anticipate that, in a real-world setting, more women would, and could, benefit from early cardiovascular screening and intervention when they have experienced preeclampsia.

\section{Comparison with other studies}

Two Dutch studies showed the potential benefits of early hypertension and metabolic syndrome detection, including medication and/or lifestyle intervention, in women with a history of preeclampsia. ${ }^{20,21}$ These studies concluded that CVD prevention in women with preeclampsia is likely to be cost-effective or may save costs without affection quality of life for the first 10-20 years. The difference in results and conclusions of the current study and the two previous studies may be related to the use of medication as an intervention strategy following CVD screening. As an intervention targeting a change in lifestyle (eating, drinking, smoking and physical activity habits) takes more time and it is more expensive, than starting a (relatively cheap) drug.

Both previous studies use blood pressure $(\geq 140 / 99 \mathrm{~mm} \mathrm{Hg}$ ) for treatment selection whereas the treatment selection of our study was risk-based. Furthermore, the published studies used a Markov model with a number of 'health states' with fixed transitions between states, whereas we used an individual patient-level model. This provided the opportunity to include CVD risk factors, simulated events and outcomes on an individual level which moves closer to individualised care. For the current study, all individual CVD risk factors were combined in one risk estimate and the change in expected risk was modelled over time. For future research, it is possible to further detail individual risk assessment by also incorporating the assessed CVD risk factor levels per individual. Furthermore, the use of real-world follow-up data of women at 10-20 years post-preeclampsia to estimate the CVD risks and subsequent correlation between risk profiles likely has led to more accurate and realistic results, compared with studies making assumptions on risk development over time.

\section{Strengths}

The strength of this study is the incorporation of actual risk factor data from women who underwent 
cardiovascular screening at several time points after preeclampsia. These data gave insight in the risk distribution among women with preeclampsia for different age categories. Furthermore, these data were used to estimate the correlation between 10-year risk estimates within women over time. A micro-simulation model was used to assess the long-term benefits of CVD risk screening combined with risk-based lifestyle advice in young women. The use of a model with a lifetime horizon is important, as age is a key factor in development of CVD. Moreover, the first manifestation of CVD may take two to four decades following preeclampsia. ${ }^{42} \mathrm{We}$ postulate that this model, with simple adjustments, can be applied to assess the potential benefits of early CVD risk screening combined with any subsequent risk-based intervention in other populations with (female) specific risk factors, such as women with polycystic ovarian syndrome (PCOS) or premature ovarian insufficiency (POI) or migraine with aura. ${ }^{43,44}$ Use of such models may provide information to make evidence-based guidelines and decisions for establishing cardiovascular prevention programmes for women with a medical history, while the evidence of intervention studies in these specific female subgroups is still lacking.

\section{Limitations}

Since evidence on several parameters within the model was lacking, certain assumptions had to be made and extrapolation was required. To properly reflect the uncertainty in the parameters, we allowed relatively wide distributions for most parameters and incorporated expert opinions on behaviour, risks and benefits of interventions in this specific group of women. Also, data on CVD risk after 80 years of age was lacking and this risk was therefore kept constant beyond this age.

Furthermore, we considered only preventive intervention of lifestyle changes for both young and older women. This approach may not be realistic in clinical practice since lifestyle modification is known to be difficult to achieve and the effectiveness is rather low. ${ }^{29,45,46}$ Additionally, lifestyle interventions were not combined with any drug therapies, such as lipidlowering or antihypertensive medication. However, as women were young during the post-partum risk evaluation, the use of life long drug therapy from a young age onwards is perhaps unrealistic. Nevertheless, some young women may be willing to take medication when becoming aware of their CVD risk after having suffered from preeclampsia. ${ }^{47}$ For example, the proportion of women that answered 'yes' to the question 'Do you have a prescription of preventive medication?' in the initial cardiovascular screening is around 19\% (see Supplemental Material Appendix C). Additionally, a notable proportion had health complaints due to hypertension shortly after pregnancy, making it more likely that they would be willing to use medical therapy, even at their young age. ${ }^{47,48}$ Further research on the optimal (age-dependent) combination of lifestyle interventions, preventive medication or other preventive interventions (e.g. self-monitoring with e-health applications), and the actual adherence to these interventions should be conducted. ${ }^{49}$

Recent CVD preventive guidelines have supported treatment of young individuals even though evidence from randomised or cohort studies for these implementations is not yet available. ${ }^{50}$ Taking these possibilities into consideration, the assumption that women in our 'no screening' scenario are not identified, or treated, before the age of 60 years may lead to an underestimation of the benefits of usual care in reality. This needs to be evaluated further and may need to be taken in to account when performing similar research in the future.

Also, we estimated CVD risk with FRS, which might not be suitable for young women with previous preeclampsia. Age is a strong contributor to this score and although women with previous preeclampsia develop CVD as soon as 10 years earlier, FRS is often not raised above the indicative $10 \%$ threshold soon after pregnancy. ${ }^{11,12}$ Unfortunately, there is no CVD risk score available that includes a (complicated) obstetric history as predictor.

Additionally, we used data from women with both late and early onset preeclampsia for this study. Although this gives a relevant overview of women with previous preeclampsia, this may underestimate the possible benefits of screening for women with a severe, or early, phenotype. Results from our analysis can therefore also not directly be extrapolated to women with other pregnancy complications (or specific phenotypes of preeclampsia), as the preventive effects are likely to differ in those women. Lastly, we were not able to consider comorbidities or the occurrence of other diseases, like auto-immune disorders or impaired memory, associated with preeclampsia and affecting the outcome and quality of life in these women. ${ }^{51}$

\section{Conclusion}

Our model-based impact assessment demonstrates that CVD risk screening combined with risk-based lifestyle interventions (without preventive treatment initiation) to prevent CVD in women with a history of preeclampsia is not cost-effective. This study shows that for establishing a beneficial cardiovascular prevention program for women starting early after experiencing preeclampsia, a more effective intervention or combination of interventions may be more realistic. 


\section{Acknowledgements}

The consortium members consist of (in alphabetical order): $\mathrm{Y}$ Appelman $^{1}$, S Baart ${ }^{2,3}$, L Benschop ${ }^{2,3}$, E Boersma ${ }^{2}$, L Brouwers $^{3,4}$, RPJ Budde ${ }^{2}$, SC Cannegieter ${ }^{5}, \mathrm{~V} \mathrm{Dam}^{3,6}$, R Eijkemans $^{6}$, BCJM Fauser ${ }^{4}$, MD Ferrari ${ }^{5}$, A Franx ${ }^{4}$, CJM de Groot ${ }^{1}$, MN Gunning ${ }^{3,4}$, A Hoek ${ }^{7}$, H Koffijberg ${ }^{6,8}$, MPH Koster ${ }^{2}$, MC Kruit ${ }^{5}$, GR Lagerweij ${ }^{3,6}$, CB Lambalk ${ }^{1}$, JSE Laven ${ }^{2}$, KM Linstra ${ }^{2,3,5}$, A van der Lugt ${ }^{2}$, AHEM Maas9, A Maassen van den Brink ${ }^{2}$, C Meun ${ }^{2,3}$, S Middeldorp ${ }^{10}$, KGM Moons ${ }^{6}$, BB van Rijn ${ }^{2,4}$, JE Roeters van Lennep ${ }^{2}$, JW Roos-Hesselink ${ }^{2}$, LJJ Scheres ${ }^{3,10}$, YT van der Schouw ${ }^{6}$, EAP Steegers ${ }^{2}$, RPM Steegers-Theunissen ${ }^{2}$, GM Terwindt ${ }^{5}$, BK Velthuis ${ }^{4}$, MJH Wermer ${ }^{5}$, B Zick ${ }^{2,5}$, GA Zoet. ${ }^{3,4}$

${ }^{1}$ Vrije Universiteit Medical Center, the Netherlands; ${ }^{2}$ Erasmus University Medical Center, the Netherlands; ${ }^{3}$ Netherlands Heart Institute, the Netherlands; ${ }^{4}$ University Medical Center Utrecht, the Netherlands; ${ }^{5}$ Leiden University Medical Center, the Netherlands; ${ }^{6}$ Julius Center, University Medical Center, the Netherlands; ${ }^{7}$ University Medical Center Groningen, the Netherlands; ${ }^{8}$ University of Twente, the Netherlands; ${ }^{9}$ Radboud University Medical Center, the Netherlands; ${ }^{10}$ Academic Medical Center Amsterdam, the Netherlands.

\section{Author contribution}

BBvR, GAdW, GRL, HK, KGMM and $\mathrm{LBr}$ contributed to the conception and design of the work. AHEMM, AF, BBvR, GAdW, GRL, HK, JERvL, KGMM, LBe, LBr, and MJHW contributed to the acquisition and interpretation of data for the work, and GRL and HK contributed to the analysis of the data. GAdW, GRL, HK and $\mathrm{LBr}$ drafted the manuscript. AHEMM, AF, BBvR, GAdW, GRL, HK, JERvL, $\mathrm{KGMM}, \mathrm{LBe}, \mathrm{LBr}$ and MJHW critically revised the manuscript and gave final approval and agreed to be accountable for all aspects of work ensuring integrity and accuracy.

\section{Declaration of conflicting interests}

The author(s) declared no potential conflicts of interest with respect to the research, authorship and/or publication of this article.

\section{Funding}

The author(s) disclosed receipt of the following financial support for the research, authorship and/or publication of this article: This research forms part of the CREW NHS project, grand number 2013T083, co-funded by the Dutch Heart Foundation. The Dutch Heart Foundation had no role in the collection, analysis and interpretation of data, nor in the decision to submit the article for publication. MJHW is supported by a personal grant from the Netherlands Organization for Scientific Research (NWO) (VIDI-91717337).

\section{References}

1. World Health Organization. Women's health. Fact sheet no. 334, http://www.who.int/mediacentre/factsheets/fs334/ en/ (2013, accessed 11 July 2017).
2. World Health Organization. Cardiovascular diseases (CVDs). Factsheet, http://www.who.int/mediacentre/ factsheets/fs317/en/ (2017, accessed 11 July 2017).

3. Yusuf S, Hawken S, Ounpuu S, et al. Effect of potentially modifiable risk factors associated with myocardial infarction in 52 countries (the INTERHEART study): Casecontrol study. Lancet 2004; 364: 937-952.

4. Mol BWJ, Roberts CT, Thangaratinam S, et al. Preeclampsia. Lancet 2016; 387: 999-1011.

5. Zoet GA, Benschop L, Boersma E, et al. Prevalence of subclinical coronary artery disease assessed by coronary computed tomography angiography in 45- to 55-year-old women with a history of preeclampsia. Circulation 2018; 137: 877-879.

6. Wu P, Haththotuwa R, Kwok CS, et al. Preeclampsia and future cardiovascular health: A systematic review and meta-analysis. Circ Cardiovasc Qual Outcomes 2017; 10: 9 .

7. Bellamy L, Casas JP, Hingorani AD, et al. Pre-eclampsia and risk of cardiovascular disease and cancer in later life: Systematic review and meta-analysis. BMJ 2007; 335: 974.

8. Brown MC, Best KE, Pearce MS, et al. Cardiovascular disease risk in women with pre-eclampsia: Systematic review and meta-analysis. Eur J Epidemiol 2013; 28: 1-19.

9. Leon LJ, McCarthy FP, Direk K, et al. Preeclampsia and cardiovascular disease in a large UK pregnancy cohort of linked electronic health records: A CALIBER study. Circulation 2019; 140: 1050-1060.

10. Sciomer S, Moscucci F, Maffei S, et al. Prevention of cardiovascular risk factors in women: The lifestyle paradox and stereotypes we need to defeat. Eur J Prev Cardiol 2019; 26: 609-610.

11. Behrens I, Basit S, Melbye M, et al. Risk of post-pregnancy hypertension in women with a history of hypertensive disorders of pregnancy: Nationwide cohort study. BMJ 2017; 358: j3078.

12. Engeland A, Bjorge T, Klungsoyr K, et al. Preeclampsia in pregnancy and later use of antihypertensive drugs. Eur J Epidemiol 2015; 30: 501-508.

13. Sattar N and Greer IA. Pregnancy complications and maternal cardiovascular risk: Opportunities for intervention and screening? BMJ 2002; 325: 157-160.

14. Nijdam ME, Timmerman MR, Franx A, et al. Cardiovascular risk factor assessment after pre-eclampsia in primary care. BMC Fam Pract 2009; 10: 77.

15. Bushnell $\mathrm{C}$ and McCullough L. Stroke prevention in women: Synopsis of the 2014 American Heart Association/American Stroke Association guideline. Ann Intern Med 2014; 160: 853-857.

16. Heida KY, Bots ML, de Groot CJ, et al. Cardiovascular risk management after reproductive and pregnancyrelated disorders: A Dutch multidisciplinary evidencebased guideline. Eur J Prev Cardiol 2016; 23: 1863-1879.

17. Piepoli MF, Hoes AW, Agewall S, et al. 2016 European guidelines on cardiovascular disease prevention in clinical practice: The Sixth Joint Task Force of the European Society of Cardiology and Other Societies on Cardiovascular Disease Prevention in Clinical Practice (constituted by representatives of 10 societies and by 
invited experts)Developed with the special contribution of the European Association for Cardiovascular Prevention \& Rehabilitation (EACPR). Eur Heart $J$ 2016; 37: 2315-2381.

18. van Rijn BB, Nijdam ME, Bruinse $\mathrm{HW}$, et al. Cardiovascular disease risk factors in women with a history of early-onset preeclampsia. Obstet Gynecol 2013; 121: 1040-1048.

19. Lagerweij GR, de Wit GA, Moons KG, et al. A new selection method to increase the health benefits of CVD prevention strategies. Eur J Prev Cardiol 2018; 25: 642-650.

20. Drost JT, Grutters JP, van der Wilt GJ, et al. Yearly hypertension screening in women with a history of preeclampsia: A cost-effectiveness analysis. Neth Heart $J$ 2015; 23: 585-591.

21. van Baaren GJ, Hermes W, Franx A, et al. Cost-effectiveness analysis of cardiovascular risk factor screening in women who experienced hypertensive pregnancy disorders at term. Pregnancy Hypertens 2014; 4: 264-270.

22. Lagerweij GR. Quantification of the impact of prediction models: Model first trial later. Utrecht: Utrecht University, 2019.

23. Statistics Netherlands. Birth; key figures. https://opendata.cbs.nl/statline/\#/CBS/en/dataset/37422eng/ table?ts $=1578657567949$ (2019).

24. Bouthoorn SH, Gaillard R, Steegers EA, et al. Ethnic differences in blood pressure and hypertensive complications during pregnancy: The Generation R study. Hypertension 2012; 60: 198-205.

25. D'Agostino Sr RB, Vasan RS, Pencina MJ, et al. General cardiovascular risk profile for use in primary care: The Framingham Heart Study. Circulation 2008; 117: $743-753$.

26. Van Buuren $\mathrm{S}$ and Groothuis-Oudshoorn K. mice: Multivariate Imputation by Chained Equations in R. Journal of Statistical Software 2011; 45: 67.

27. NHG. Nederlands Huisartsen genootschap. Cardiovascular risk management. https://www.nhg.org/ standaarden/volledig/cadriovasculair-risicomanagement (2012).

28. Mosca L, Benjamin EJ, Berra K, et al. Effectivenessbased guidelines for the prevention of cardiovascular disease in women-2011 update: A guideline from the American Heart Association. J Am Coll Cardiol 2011; 57: 1404-1423.

29. Berks D, Hoedjes M, Raat H, et al. Risk of cardiovascular disease after pre-eclampsia and the effect of lifestyle interventions: A literature-based study. BJOG 2013; 120 : 924-931.

30. Beulens JW, Monninkhof EM, Verschuren WM, et al. Cohort profile: The EPIC-NL study. Int $J$ Epidemiol 2010; 39: 1170-1178.

31. Lagerweij GR, de Wit GA, Moons KGM, et al. Predicted burden could replace predicted risk in preventive strategies for cardiovascular disease. J Clin Epidemiol 2018; 93 : 103-111.

32. Mommersteeg PM, Drost JT, Ottervanger JP, et al. Long-term follow-up of psychosocial distress after early onset preeclampsia: The Preeclampsia Risk EValuation in
FEMales cohort study. J Psychosom Obstet Gynaecol 2016; 37: 101-109.

33. Stramrood CA, Wessel I, Doornbos B, et al. Posttraumatic stress disorder following preeclampsia and PPROM: A prospective study with 15 months follow-up. Reprod Sci 2011; 18: 645-653.

34. Ara R and Brazier J. Health related quality of life by age, gender and history of cardiovascular disease: results from the Health Survey for England. Sheffield: The University of Sheffield, 2009, p. 28.

35. Zhou X, Niu JM, Ji WJ, et al. Precision test for precision medicine: Opportunities, challenges and perspectives regarding pre-eclampsia as an intervention window for future cardiovascular disease. Am J Transl Res 2016; 8: 1920-1934.

36. National Institute for Health and Care Excellence (NICE). Lipid modification: Cardiovascular risk assessment and the modification of blood lipids for the primary and secondary prevention of cardiovascular disease. Clinical Guideline; Methods, Evidence and Recommendations. London: NICE, 2014, p. 302.

37. Greving JP, Visseren FL, de Wit GA, et al. Statin treatment for primary prevention of vascular disease: Whom to treat? Cost-effectiveness analysis. BMJ 2011; 342: d1672.

38. Stam-Slob MC, van der Graaf Y, de Boer A, et al. Costeffectiveness of PCSK9 inhibition in addition to standard lipid-lowering therapy in patients at high risk for vascular disease. Int J Cardiol 2018; 253: 148-154.

39. Guideline for economic evaluations in healthcare. The Netherlands: Health Care Insurance Board. 2016.

40. Strong M, Oakley JE and Brennan A. Estimating multiparameter partial expected value of perfect information from a probabilistic sensitivity analysis sample: A nonparametric regression approach. Med Decis Making 2014; 34: 311-326.

41. Wilson EC. A practical guide to value of information analysis. Pharmacoeconomics 2015; 33: 105-121.

42. Perk J, De Backer G, Gohlke H, et al. European guidelines on cardiovascular disease prevention in clinical practice (version 2012). The Fifth Joint Task Force of the European Society of Cardiology and Other Societies on Cardiovascular Disease Prevention in Clinical Practice (constituted by representatives of nine societies and by invited experts). Eur Heart $J$ 2012; 33: 1635-1701.

43. de Groot PC, Dekkers OM, Romijn JA, et al. PCOS, coronary heart disease, stroke and the influence of obesity: A systematic review and meta-analysis. Hum Reprod Update 2011; 17: 495-500.

44. Roeters van Lenne JE, Heida KY, Bots ML, et al. Cardiovascular disease risk in women with premature ovarian insufficiency: A systematic review and meta-analysis. Eur J Prev Cardiol 2016; 23: 178-186.

45. Statistics Netherlands. Health, lifestyle, health care use and supply, causes of deaths; key figures. Statline, https://opendata.cbs.nl/statline/\#/CBS/en/dataset/ 81628ENG/table?ts $=1578657349051$ (2019).

46. Paauw ND, Luijken K, Franx A, et al. Long-term renal and cardiovascular risk after preeclampsia: Towards 
screening and prevention. Clin Sci (Lond) 2016; 130: 239-246.

47. Maas AH. Screening after hypertensive pregnancy disorders: She can do best. Eur J Prev Cardiol 2017; 24: 1733-1734.

48. Benschop L, Duvekot JJ, Versmissen J, et al. Blood pressure profile 1 year after severe preeclampsia. Hypertension 2018; 71: 491-498.

49. Saner $\mathrm{H}$ and van der Velde E. eHealth in cardiovascular medicine: A clinical update. Eur J Prev Cardiol 2016; 23 : S5-S12.
50. Mancia G, et al. 2013 ESH/ESC guidelines for the management of arterial hypertension: The Task Force for the management of arterial hypertension of the European Society of Hypertension (ESH) and of the European Society of Cardiology (ESC). J Hypertens 2013; 31: 1281-1357.

51. Williams D. Long-term complications of preeclampsia. Semin Nephrol 2011; 31: 111-122. 